\title{
Prediction of Contra-Rotating Open Rotor broadband noise in isolated and installed configurations
}

\author{
T. Nodé-Langlois ${ }^{1}$, F. Wlassow ${ }^{2}$, V. Languille ${ }^{3}$, Y. Colin², B. Caruelle ${ }^{2}$ \\ Airbus Operations SAS, 31060 Toulouse, France \\ J. Gill ${ }^{4}$, X. Chen ${ }^{5}$, X. Zhang ${ }^{6}$ \\ University of Southampton, Hampshire, SO17 1BJ, UK. \\ A.B Parry ${ }^{7}$ \\ Rolls-Royce plc, Derby, DE248BJ, UK
}

\begin{abstract}
Broadband noise is a significant part of the noise emitted by contra-rotating open rotors. Several noise sources can contribute to the total broadband sound field, with the most dominant ones probably being trailing edge noise, rotor-wake interaction noise and pylon-wake interaction noise. This paper addresses the prediction of these noise sources using analytical models based on Amiet's flat plate airfoil theory and also to empirical turbulence models, fed by input data extracted from steady and unsteady CFD RANS simulations. The models are assessed against wind tunnel tests of Rolls-Royce's rig 145 (build 1) conducted at the DNW anechoic open jet test facility using Rolls-Royce blades and Airbus pylons. The study showed promising results in terms of the ability of the models to predict acoustic power spectrum shapes, peak frequencies and absolute levels. The effects of changes in thrust on broadband wake-interaction noise are well reproduced. However, the models significantly underestimate the effect of thrust on trailing edge noise and the effect of rotational velocity on pylon interaction noise.
\end{abstract}

\section{Nomenclature}

$\begin{array}{ll}\text { AI } & =\text { Airbus } \\ \text { ANTC } & =\text { Airbus Noise Technology Centre } \\ C F D & =\text { Computational Fluid Dynamics } \\ C R O R & =\text { Counter Rotating Open Rotors } \\ D N W & =\text { German-Dutch wind tunnels } \\ \text { LES } & =\text { Large Eddy Simulation } \\ P W L & =\text { Acoustic Power Level } \\ r p m & =\text { Revolutions per minute } \\ R R & =\text { Rolls-Royce } \\ S O R & =\text { Single open rotor }\end{array}$

\section{Introduction}

S ince the publication of the ACARE goals, the commercial, political and environmental pressure to reduce $S$ carbon dioxide emissions has increased considerably. To address these challenges, aerospace companies are once again looking at counter rotating open-rotor (CROR) technology. The advantage of such a technology is that it has potential for significant fuel savings ${ }^{1}$. However, one of the main challenges is to significantly reduce noise levels (both cabin and far field) in order to achieve certification against more stringent noise targets.

\footnotetext{
${ }^{1}$ Acoustic Engineer, Numerical Acoustics Group, 316 Route de Bayonne, thomas.node-langlois@airbus.com

${ }^{2}$ Acoustic Engineer, Numerical Acoustics Group

${ }^{3}$ Intern, Numerical Acoustics Group (now ATR company)

${ }_{5}^{4}$ EngD student, Airbus Noise Technology Centre, Faculty of Engineering

${ }^{5}$ Research fellow, Airbus Noise Technology Centre, Faculty of Engineering

${ }^{6}$ Airbus Professor of Aircraft Engineering, Airbus Noise Technology Centre, Faculty of Engineering, Associated fellow, AIAA.

${ }^{7}$ Engineering Specialist - Aerodynamics, Rolls-Royce plc, PO Box 31.
} 
Very significant efforts have been put over the last decade in the research community to develop prediction methods for CROR tonal noise, as this was identified as a major hurdle during the CROR research programs executed during the 80s. Now that tonal noise has been significantly reduced thanks to, for instance, advanced blade design $^{2}$ and pylon blowing technology ${ }^{27}$, broadband noise constitutes a significant part of the noise emitted by CRORs. Its accurate prediction has become a requirement to support design optimization of the CROR-powered aircraft.

One of the first, and most significant works, on open rotor broadband noise modelling was made by Paterson and Amiet ${ }^{14}$ in the late 70s on the ingestion of turbulence by a rotor using the simple approach proposed a few years before by Amiet $^{10}$, which approximates the circular motion of the blades by a series of translations of the blades over an infinitesimally short distance. The same approach was then used by Schlinker and Amiet ${ }^{13}$ for the prediction of helicopter trailing edge noise.

A solution for the exact rotational motion in the Ffowcs-Williams Hawking equations was proposed for the first time by Kim and George ${ }^{18}$ for trailing edge noise. However, they approximated the unsteady blade loading along the chord as a point force. This simplification was enhanced later by Zhou and $\operatorname{Joseph}^{33}$ (2004) for open propellers using Amiet's isolated airfoil response function ${ }^{8,9}$ extended to take into account the contribution of skewed gusts, and then extended by Blandeau ${ }^{3}$ for contra-rotating open rotors. A significant contribution for the trailing edge noise prediction was achieved by Roger and Moreau ${ }^{35}$, who introduced a correction for the back-scattering of the leading edge. Other applications have been made for instance by Rozenberg for low speed fans, and by Glegg ${ }^{28}$ for wind turbines.

The specific case of the interaction of the wake of the front rotor of a CROR with its rear rotor was tackled by Blandeau $^{3}$ and an adaptation of this theory to model the interaction between the wake of the pylon supporting the engine and the front rotor was developed by Kingan ${ }^{34}$.

\section{Problem description and study objectives}

\section{A. CROR noise sources}

Contra-rotating open rotors feature broadband noise sources rather similar to those that can be found in a turbofan engine. They will differ in their radiation characteristics due to the absence of the nacelle, such that the duct mode radiation of the turbofan will be replaced by free field radiation. One might normally expect more efficient noise radiation from a CROR since no duct cut-off phenomenon will occur. However, the unsteady aerodynamic source mechanisms will be essentially the same and the effect of radiation efficiency on a CROR is also closely analogous to that on a ducted configuration, as discussed by Peake $\&$ Parry $^{45}$. These source mechanisms can be split into two categories: rotor alone noise sources and interaction noise sources.

The possible rotor alone noise sources are trailing edge noise, tip noise and flow separation noise. The trailing edge noise is produced by the deformation of the turbulent boundary layer vortices when they pass over the rotor trailing edge to satisfy continuity between pressure side and suction side static pressures. (This criterion is referred to as the Kutta condition and its application in unsteady flow is discussed by Crighton ${ }^{46}$ ). The temporal evolution of the vorticity just downstream of the trailing edge leads to efficient noise sources thanks to the diffraction of the acoustic waves on the trailing edge of the blade. Rotor tip noise is produced by the complex three-dimensional turbulent flow around the tip of the rotor. The mechanism is similar to the trailing edge noise but implies diffraction of the turbulent vortices by the rotor tip edge and the highly three dimensional characteristic of the flow leads to more complex phenomenology. The weight of this source versus the trailing edge noise is currently unknown. One can argue that only a limited portion of the blade is involved in this noise mechanism, but this is counterbalanced by the highest rotational speed on the blade. Finally, flow separation noise can be generated by large and unsteady flow separation on the blades. For high efficiency rotor blade designs, such separation is not expected when operating in design conditions (take-off, cruise), but it should be avoided as much as possible in off-design conditions, such as approach, in order to not significantly impact the certification noise margin.

There are many possible sources of interaction noise between the two rotors. The most significant one is due to the interaction of the front rotor turbulent wakes with the rear rotor blades. Turbulent wakes induced by the boundary layers of the front rotor are propagating in a helical pattern towards the leading edge of the rear rotor. Turbulent velocity fluctuations in the wake lead to stochastic variation of the blade incidence, hence causing blade loading fluctuations and efficient dipolar noise emission. Similarly to the rotor alone discussion, a special case of this noise source is the interaction of the front rotor tip vortex turbulence with the tip of the rear blade. Again, the highly three dimensional behaviour of the flow will make this interaction more complex than other interaction noise 
sources, not just because of the nature of the unsteady velocity field incident on the downstream row but also because of the unsteady response of the downstream blades for which the three-dimensional tip-edge effect is likely to have an important role. This noise source is generally avoided by cropping the rear blade (reduced tip diameter), but its effects may remain in part, especially when the upstream inflow to the rotors features incidence. Another area of intense turbulence is the boundary layer of the nacelle. It will interact with the root of the front and rear blade and may constitute a significant additional source whose specificity will be the anisotropy of the turbulent structures due to their longitudinal stretching during propagation along the nacelle walls. However, the significantly lower relative speed of the blade profiles at the root compared to the blade tip area may limit the strength of this source, though the additional effects of radiation efficiency may enable it to contribute to the total broadband noise at certain frequencies. Finally, the ingestion of atmospheric turbulence by the rotor may result in noise generation when the turbulence interacts with the fan, however this is likely to constitute a small source in standard atmosphere conditions and when the CROR is in flight (rather than stationary), see Robison \& Peake ${ }^{47}$ and Robison ${ }^{48}$.

All of the discussed sources are present on an isolated CROR. However, when the CROR is installed on the aircraft in a pusher configuration, with the pylon being located upstream of the front rotor, an additional interaction noise source will be generated. This is caused by the impingement of the pylon wake turbulent vortices on the leading edge of the front rotor and, to a lesser extent, on the leading edge of the rear rotor after the pylon wake has interacted with, and been deformed by, the front rotor. Also, pylon trailing edge noise may be considered as a broadband noise source in its own right; the absence of rotational velocity in this case will limit the aerodynamic strength of this source though, as mentioned previously, the radiation efficiency is governed by a combination of speed and frequency and receiver location so the source may contribute over part of the spectrum and/or directivity..

The potential presence of all those sources on a CROR makes the prediction of the CROR total broadband noise a significant challenge, increased by the fact that generally those sources cannot be easily separated experimentally as they produce noise in a similar frequency range. However, a big advantage compared to turbofans is the possibility to run a clean single rotor test in which the possible broadband sources are limited to rotor alone noise and nacelle boundary layer interaction noise.

\section{B. Objectives of the study}

The objective of the present study was to assess noise prediction models available in the literature as comprehensively as possible, and therefore to find the sources which are expected to be dominant in CRORs. For a single rotor configuration, the trailing edge noise has typically been assumed as the dominant source, while for a CROR configuration the trailing edge noise, rotor-wake interaction noise and pylon-wake interaction noises are all considered. Although it has not been demonstrated that the other possible sources can be neglected (such as tip noise, etc.), it is commonly accepted that the sources we have selected are significant and must be accounted for in a first approximation.

This paper will first explain the validation test cases used in this study, followed by a description of the prediction models for trailing edge noise, rotor-wake interaction noise and pylon-wake interaction noise - including the generation of input data to those noise models from both CFD computations and empirical models. Finally, an assessment of the models on the selected test cases will be presented.

\section{Experimental set-up}

Wind tunnel tests were performed in 2008 jointly by Rolls-Royce (RR) and Airbus (AI) in the German-Dutch DNW anechoic open jet wind tunnel in the Netherlands. The tests were undertaken as part of the EU's DREAM programme. The rig (numbered Rig145 build 1) was designed by RR, the blades were produced by Dowty to RR's specification, while the pylons were designed and manufactured by AI. Whilst the detailed tests included a number of different isolated and installed configurations, including different blade numbers/designs and different pylon designs/arrangements, the configurations of interest in this study are: front rotor alone, isolated CROR, and installed CROR with an upstream pylon. These three configurations will allow us to separate the noise sources into rotor alone, rotor-rotor interaction and pylon induced noise components. The front rotor alone pitch setting was defined such the that same thrust was obtained at take-off rotational speeds for both the isolated rotor test and for the front rotor of the CROR configuration, thus targeting a similar blade loading.

The front rotor alone configuration will be used in the following study to assess trailing edge noise models. Then the rotor-wake interaction noise model will be assessed in comparison to the noise measured for the CROR configuration. This measurement will be obtained by subtracting an estimation of front and rear rotor alone noise 
(based on the front rotor alone measurement) from the total CROR noise measurement. Finally, pylon induced noise will be obtained by the difference between installed CROR and isolated CROR data.

The experimental data used in this study will be based on the out-of-flow microphones located in the quiescent medium outside of the wind tunnel jet on the wall of the facility. Correction for shear layer refraction is made based on Schlinker \& Amiet ${ }^{12}$. Details on the measured narrow-band spectra and the observed installation effects were published by Ricouard ${ }^{27}$.

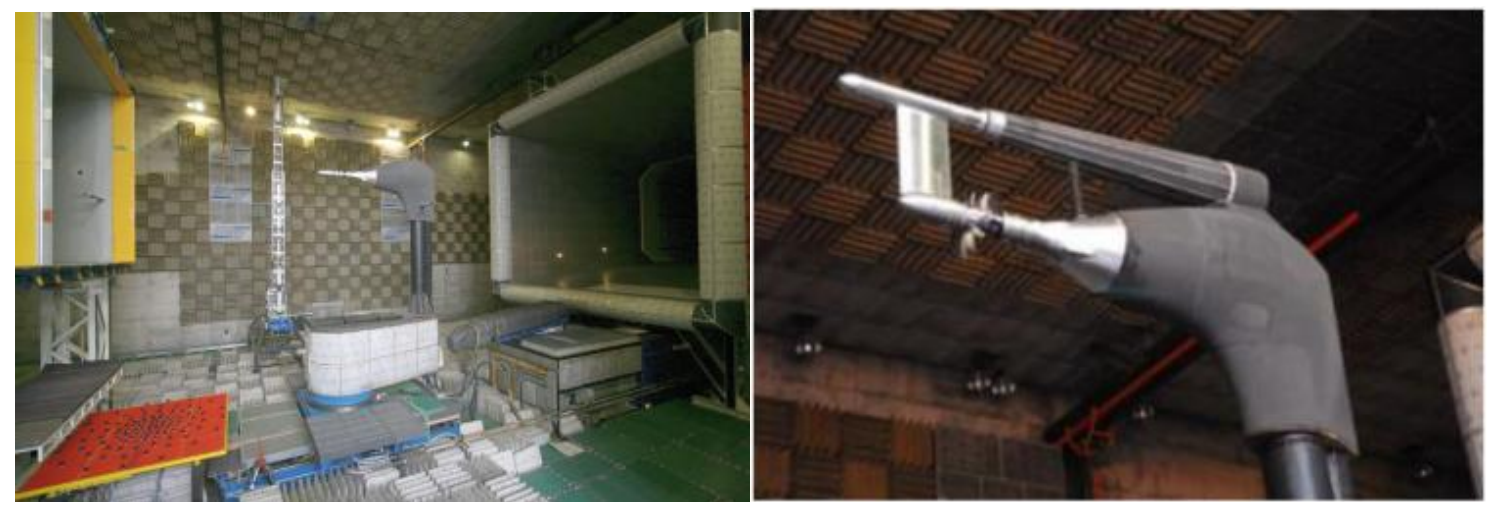

Figure 1: Rig145 in DNW Wind-tunnel facility

\section{Description of the prediction methods}

All the models that will be described in the following sections are implemented in a Matlab software called BoB ("Broadband noise of Open rotor Blades") which has been jointly developed by the Airbus Noise Technology Centre (University of Southampton) and Airbus Company. It is a modular analytical software dedicated to open rotor broadband noise prediction, whose input data can be obtained either by embedded empirical models or by results from CFD post-processing.

\section{A. Trailing edge noise}

The trailing edge noise model is based on the work of Blandeau ${ }^{3,4}$ with a strip theory to account for the spanwise variations of aerodynamic quantities and blade geometry. The diffraction of the boundary layer turbulent vortices by the trailing edge is modelled using the isolated flat plate airfoil theory from Amiet ${ }^{8,9}$ enhanced by Roger and Moreau $^{35}$ to account for skewed gusts. Rotating dipoles are then used to compute the radiated noise field according to Ffowcs-Williams-Hawking theory ${ }^{15}$ with an approach similar to Kim and George ${ }^{18}$, however fully accounting for the non-compactness of the sources. The final expression for the power spectral density of the acoustic pressure radiated by a single strip on an observation point of polar coordinate $\left(r_{0}, \theta_{0}, \varphi_{0}\right)$ reads:

$$
\begin{aligned}
S_{p p}^{T E}\left(r_{0}, \theta_{0}, \omega\right)=\frac{B \Delta r}{2 \pi}\left(\frac{b}{r_{0} \sqrt{1-M_{x}^{2} \cdot \sin ^{2} \theta_{0}}}\right)^{2} . & \\
& \sum_{l=-\infty}^{+\infty} D_{l}\left(\theta_{0}, \alpha, \omega\right) \cdot l_{r}\left(k_{r}=0, k_{X, l}\right) \cdot \Phi_{p p}\left(k_{X, l} \cdot U_{c}\right) \cdot\left|\mathcal{L}^{T E}\left(k_{r}=0, k_{X, l}, \kappa_{l}\right)\right|^{2}
\end{aligned}
$$

where $B$ is the number of blades, $M_{x}$ the propeller infinite upstream Mach number, $\Delta r$ the strip width, $b$ its halfchord, $\alpha$ its stagger angle and $U_{c}$ the convection velocity of the turbulent eddies in the boundary layer just upstream to the trailing edge. $D_{l}$ is a directivity term that depends only on the geometry and the frequency, while $\mathcal{L}^{T E}$ is an aeroacoustic coupling integral along the profile chord based on Amiet's model ${ }^{3} . \kappa_{l}$ is an aeroacoustic coupling wavenumber and $k_{X, l}$ the chordwise turbulent wavenumber consistent with the Doppler shifted frequency of the $l^{\text {th }}$ azimuthal acoustic mode and given by:

$$
k_{X, l}=\frac{\omega+l \Omega}{U_{c}} \quad(\Omega \text { : rotor angular speed })
$$




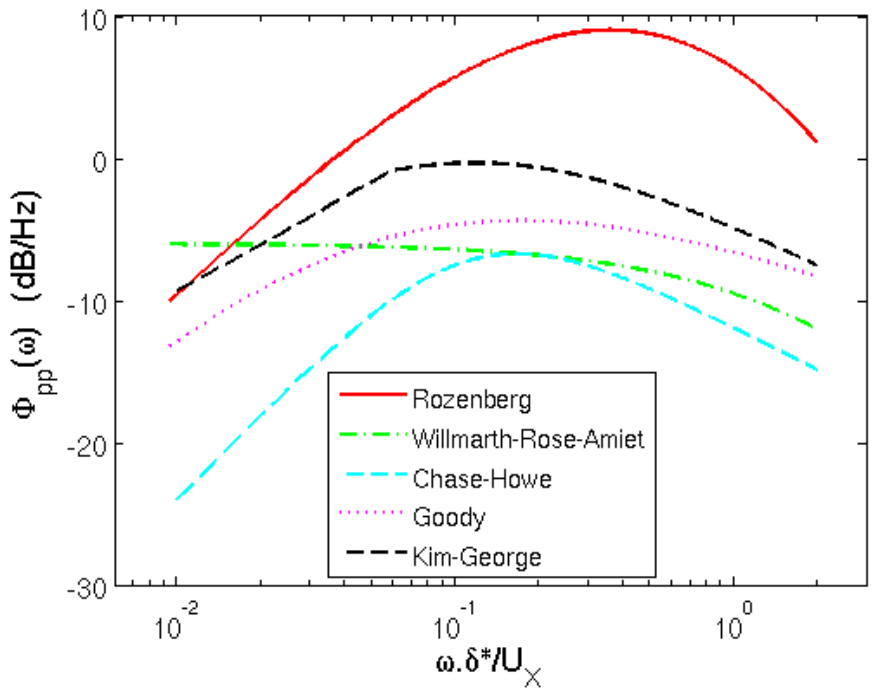

Figure 2. Surface pressure fluctuation spectrum

\begin{tabular}{|c|c|c|}
\hline & CROR blade & NACA0012 \\
\hline $\begin{array}{c}\text { External } \\
\text { Velocity }(\mathrm{m} / \mathrm{s})\end{array}$ & 249 & 65 \\
\hline $\begin{array}{l}\text { Boundary layer } \\
\text { thickness }(\mathrm{cm})\end{array}$ & 1.16 & 1.4 \\
\hline $\begin{array}{l}\text { Displacement } \\
\text { thickness }(\mathrm{cm})\end{array}$ & 0.36 & 0.24 \\
\hline $\begin{array}{l}\text { Momentum } \\
\text { thickness (mm) }\end{array}$ & 0.20 & 0.16 \\
\hline $\begin{array}{l}\text { Cole's wake } \\
\text { parameter } \Pi\end{array}$ & 1.61 & 1.56 \\
\hline $\begin{array}{l}\text { Friction } \\
\text { at wall }(\mathrm{Pa})\end{array}$ & 66 & 5.4 \\
\hline $\begin{array}{l}\text { Wall friction } \\
\text { coefficient }\end{array}$ & 0.00177 & 0.00217 \\
\hline $\begin{array}{l}\text { Max friction in } \mathrm{BL} \\
(\mathrm{Pa})\end{array}$ & 173 & - \\
\hline $\begin{array}{l}\text { Chordwise pressure } \\
\text { gradient } d p / d x\end{array}$ & 128600 & 12140 \\
\hline $\begin{array}{l}\text { Clauser parameter } \\
\beta_{c}=\theta / t_{w} \cdot(d p / d x)\end{array}$ & 3.5 & 3.5 \\
\hline $\begin{array}{c}\Phi_{p p} \text { scaling factor } \\
\tau^{2} \cdot d^{*} / U e\end{array}$ & $\begin{array}{l}0.063\left(\tau_{w}\right) \\
0.43\left(\tau_{\max }\right)\end{array}$ & $0.00108\left(\tau_{w}\right)$ \\
\hline
\end{tabular}

Table 1. Boundary layer characteristics

Finally, $\Phi_{p p}$ is the surface pressure spectral density close to the trailing edge, and $l_{r}$ is the spanwise correlation length. Thus, the model requires the assessment of three critical parameters, which are the surface pressure fluctuation spectrum, the spanwise correlation length and the convection velocity of the boundary layer turbulence. The rest of the equation is fully analytical. Unfortunately, no universal models are available to describe those three critical parameters, although a number of empirical models have been previously developed with various accuracies. For the surface pressure fluctuation spectrum, several models are available in BoB software, namely WillmarthRose-Amiet ${ }^{8}$, Chase-Howe ${ }^{16}$, Goody ${ }^{17}$, Kim-George $^{18}$ and the recent Rozenberg ${ }^{19}$ models.

Figure 2 shows the spectra obtained for the RR blade studied in this paper at $85 \%$ of span for a take-off power setting. A huge $16 \mathrm{~dB}$ difference is observed for peak level between the extreme Chase-Howe and Rozenberg models. It shall be noted here that the Rozenberg model is the only model which explicitly accounts for the adverse pressure gradient along the chord with a dedicated dependency. Among the others, only the Kim-George model accounts implicitly for adverse pressure gradient because it is based on NACA0012 airfoil experimental data, which explains its higher level compared to the other models in Figure 2. Table 1 shows that the CROR airfoil has very similar boundary layer characteristics as the NACA0012. The only significant differences are the velocity external to the boundary layer and the wall friction, that lead to very high values of the scaling factor of surface pressure fluctuation model. This means that the model is used in extrapolation of Rozenberg's validation database. Moreover, the very high level obtained with the Rozenberg model is in fact due to the use of the maximum friction coefficient in the scaling factor, instead of its wall value which shows up about three times lower in the CROR case, leading to an increase by a factor of about 9 and explaining most of the $10 \mathrm{~dB}$ difference with the Kim-George model. Again, here we are in strong extrapolation of the Rozenberg model, which was validated for a maximum friction rather close to the wall friction (ratio of 1.5 in the worst case of the CD airfoil).

The second critical parameter of the model is the convection speed of the turbulent eddies, which is classically modelled by a value of $80 \%$ of the velocity external to the boundary layer (see Amiet ${ }^{8}$ for example). However, authors like Schloemers ${ }^{22}$ and Gliebe ${ }^{21}$ have shown that this velocity shall be smaller for small eddies travelling close to the walls. More recently, Del Alamo ${ }^{38}$ performed a post-processing of DNS computations of turbulent channels. The data was averaged over two frequency ranges $\lambda / h<2$ and $\lambda / h>2$, with $h$ being the channel half-height. Del Alamo showed a reduction of the convection velocity very close to the walls for high frequency structures, down to about $40 \%$ of the mean velocity in the centre of the channel. For larger wavelengths, the convection velocity ratio at the wall was on average around $70 \%$. Del Alamo also showed that the convection velocity was almost constant across the small wavelength range: $\lambda / h<2$. When applied to the CROR case, and by 


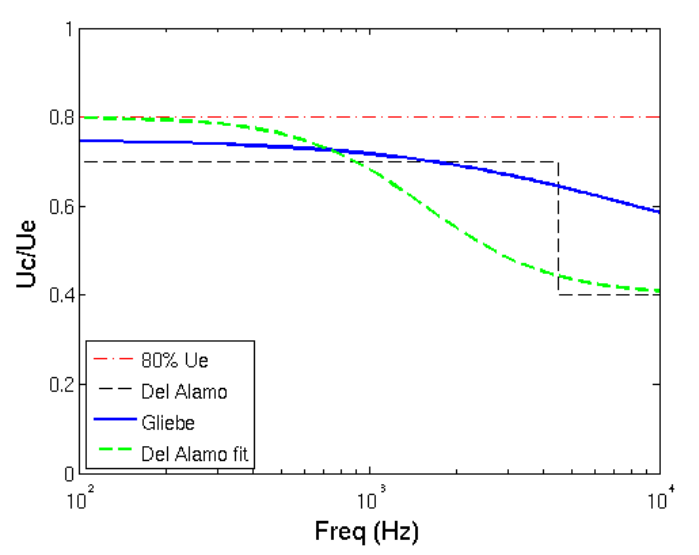

Figure 3. Convection velocity matching the channel half height to the blade boundary layer thickness, the limit $\lambda / h=2$ corresponds to a frequency of around $4.5 \mathrm{kHz}$. A comparison of the Gliebe model and Del Alamo data is shown in Figure 3. Both sets of data are similar for low frequencies while more differences are observed for the high frequency range where Del Alamo shows significantly smaller values. Although the turbulent channel data may not be fully applicable to airfoil boundary layers, a curve fit based on Del Alamo data and an asymptotic low frequency value of $80 \%$ have been drawn in order to test the sensitivity of the noise model to the convection velocity model (see Section V-A).

$$
U_{c} / U_{e}=\left(0.8+20 . S t^{2}\right) /\left(1+50 . S t^{2}\right)
$$

The spanwise correlation length is the third critical parameter of the noise model as it directly defines the spanwise extent of the correlated noise sources. Again, an empirical model shall be defined. Most authors use the well-known $\operatorname{Corcos}^{23}$ model, where the $\alpha$ coefficient has to be calibrated empirically. A value of 0.625 has been used based on Brooks and Hodgson ${ }^{39}$ for a NACA0012 airfoil. Note that it also appears to be a reasonable mean

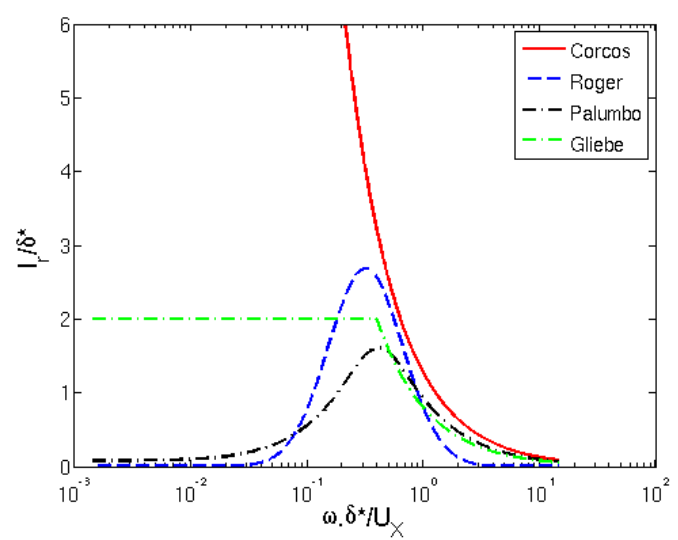

Figure 4. Spanwise correlation length value in the work of Roger and Moreau ${ }^{36}$. However, this model has incorrect low frequency asymptotic behaviour as this length unrealistically increases towards infinity. An attempt to find a physically consistent model was made by Roger $^{37}$ using a log-normal law to fit with the experimental data of low speed fans. It was based on the displacement thickness of the boundary layer $\delta^{*}$. One can also mention a similar attempt made by Palumbo ${ }^{40}$ that adjusted the model proposed by Efimsov ${ }^{41}$ to experimental data measured on a Gulfstream aircraft fuselage, but here the data is applicable to very high Reynolds number of $2-4.10^{7}$ i.e. about 50 times greater than that of the present study, and the application to the CROR case may be questionable. Also, Gliebe $^{21}$ proposed to limit the low frequency value by $2 \delta^{*}$. Figure 4 shows the comparison of the four models (using $U_{c}=80 \% U e$ for the Corcos model). The three improved models are consistent in predicting the Strouhal number where the maximum length should occur.

To feed the discussed empirical models, boundary layer parameters of the rotors shall be provided. They are obtained thanks to post-processing from CFD RANS simulations performed by Colin ${ }^{25,26}$ with the ONERA NavierStokes multi-block parallel flow solver elsA ${ }^{24}$. Since only rotor boundary layer information is necessary, steady computations using the mixing plane technique at an interface between the two rotor domain blocks provides a good estimation of the time-averaged boundary layer. It is assumed that the fluctuation of the boundary layer during rotor rotation has a negligible impact on the radiated noise. Fully automated post-processing Python scripts have been written to extract or compute the boundary layer external velocity, thickness and displacement thickness, the friction coefficient, Cole's wake parameter and the chordwise pressure gradient. (i.e. all parameters necessary to feed the empirical models described above.)

\section{B. Rotor-wake interaction noise}

The rotor-rotor interaction noise model is also based on Blandeau ${ }^{3,4}$, and also uses a strip approach. The FowcsWilliams and Hawkings analogy is used again along with the isolated flat plate theory of Amiet ${ }^{5,7}$ for the interaction of turbulent velocity gusts on a profile. The turbulent rotor wakes are assumed to be homogeneous and isotropic 
turbulence that is modulated by an infinite train of gaussian wake profiles. The final expression for the power spectral density of the acoustic pressure radiated by a single strip on an observation point at polar coordinate $\left(r_{0}, \theta_{0}, \varphi_{0}\right)$ reads:

$$
\begin{aligned}
S_{p p}^{W I}\left(r_{0}, \theta_{0}, \omega\right)=\frac{\pi B_{2}}{2}\left(\frac{\varrho_{0} B_{1} b_{2}}{r_{0} \sqrt{1-M_{x}^{2} \cdot \sin ^{2} \theta_{0}}}\right)^{2} U_{X 2} \Delta r & \\
& \sum_{n=-\infty}^{+\infty} \sum_{m=-\infty}^{+\infty} D_{m l}^{\prime}\left(\theta_{0}, \alpha_{2}, b_{w}, \omega\right) . \Phi_{w w}\left(k_{r}=0, k_{X, m n}\right) \cdot\left|\mathcal{L}^{L E}\left(k_{r}=0, k_{X, m n}^{M}, \kappa_{m n}\right)\right|^{2}
\end{aligned}
$$

where $B_{1}$ and $B_{2}$ are the number of blades of the front and rear rotor, $M_{x}$ is the propeller infinite upstream Mach number, $\Delta r$ is the rear rotor strip width, $b_{2}$ its half-chord, $\alpha_{2}$ its stagger angle and $U_{X 2}$ its relative velocity. $D_{m l}^{\prime}$ contains a directivity term that depends on the geometry and the frequency, but unlike the trailing edge case it also features an amplitude term depending on the wake half-width $b_{w}$ that accounts for the spatial extension of the wake turbulence that impinges on the rear rotor. $\mathcal{L}^{L E}$ is the aeroacoustic coupling integral along the profile chord based on Amiet's model ${ }^{3} . \kappa_{m n}$ is an aeroacoustic coupling wavenumber and $k_{X, m n}^{M}$ the chordwise turbulent wavenumber consistent with the Doppler shifted frequency of the $l^{t h}$ azimuthal acoustic mode and given by:

$$
k_{X, m n}^{M}=\frac{\omega-l \Omega_{2}}{U_{X 2}} \quad\left(\Omega_{2}: \text { rear rotor angular speed, } l=m B_{2}-n\right)
$$

Finally, $\Phi_{w w}$ is the turbulence velocity spectrum, and is usually chosen as the following Von Kármán ${ }^{6,29}$ spectrum. The Liepmann spectrum is also available in BoB and provides very similar predictions.

$$
\Phi_{w w}\left(k_{r}, k_{X}\right)=\frac{4}{9 \pi} \frac{u_{r m s}^{2}}{k_{e}^{4}} \frac{k_{r}{ }^{2}+k_{X}{ }^{2}}{\left(1+\left(k_{r}{ }^{2}+{k_{X}}^{2}\right) / k_{e}{ }^{2}\right)^{7 / 3}}
$$

with $k_{e}=\sqrt{\pi} / \Lambda \cdot \Gamma(5 / 6) / \Gamma(1 / 3)$, where $\Lambda$ is the integral length scale of the turbulence.

CFD RANS simulations are used again to feed the models. Automated post-processing Python scripts were implemented to extract the wake half-width $b_{w}$, turbulent kinetic energy $k$ and the dissipation rate $\varepsilon$ from the CFD simulations just upstream of the rear rotor leading edge. While a one-equation turbulence model such as SpalartAlmaras $^{31}$ was sufficient for the trailing edge post-processing, a two-equation model such as the k- $\omega$ SST model from Menter ${ }^{32}$ is required here to get the turbulent kinetic energy and dissipation rate as direct outputs of the CFD computation. The CFD simulation shall be accurate enough to propagate the wakes from front rotor trailing edge down to the rear rotor leading edge, accounting for potential effects from the rear rotor. The solution retained here is the unsteady RANS phase-lagged approach (chorochronic) used by Colin ${ }^{25,26}$.

The integral length scale $\Lambda$ of the turbulence may be computed from CFD data using the classical RANS formula:

$$
\Lambda=\mathrm{C}_{\Lambda} \cdot \frac{k^{3 / 2}}{\varepsilon}
$$

where $\mathrm{C}_{\Lambda}$ is a constant tied to the Kolmogorov constant. Using 1.5 for the Kolmogorov spectrum constant (see Pope ${ }^{42}$ ) yields a value of 0.39 as shown in Fedala ${ }^{43}$. However, as the integral length scale will be the primary driver for the peak frequency of the radiated noise spectrum, this constant could be empirically tuned in order to fit with measured noise.

An alternative approach is to use an empirical model such as Jurdic ${ }^{30}$ which ties this scale to a percentage of

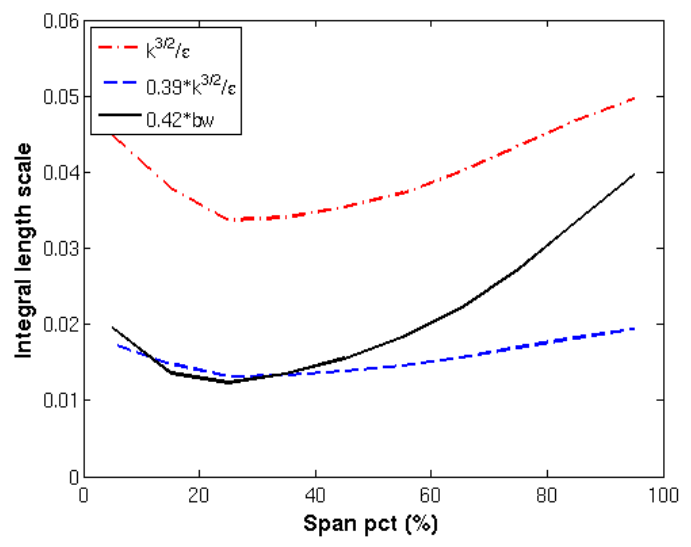

Figure 5. Wake turbulence integral length scale 
the wake half-width $b_{w}$ and which was developed from fan rig tests with hotwire measurements:

$$
\Lambda=0.42 . b_{w}
$$

A consistent result was also found by $\mathrm{Ganz}^{44}$ with a coefficient of 0.4 . A comparison between this empirical model and the RANS formula is shown in Figure 5 for a take-off power setting where the integral length scale is plotted as a function of the spanwise location. The empirical model appears to fit very well with the RANS formula with the 0.39 constant $\mathrm{C}_{\Lambda}$ for the lower half-span of the blade, while it diverges toward higher values at the tip. It is also noticeable that the RANS formula provides a much flatter profile.

\section{Pylon-wake interaction noise}

For the pylon-wake interaction noise model, there is no axisymmetry in the configuration unlike for the rotor/rotor interaction. This prevents removing the dependency on the azimuthal coordinates from the derivation. An extension of the rotor/rotor interaction model for the pylon/rotor interaction was developed by Kingan ${ }^{34}$ and has been implemented by the ANTC in the BoB software. This model accounts for the exact effect of the rotation but with the drawbacks of a significantly higher computational time, which is caused by a large summation over different modal frequencies. An alternative, and less computationally expensive, model has been developed by the ANTC that follows the approach from Amiet ${ }^{10,11}$ where the circular motion of each rotor blade profile is simplified to a series of small translations. The final expression for the power spectral density of the acoustic pressure radiated by a single strip on an observation point of polar coordinate $\left(r_{0}, \theta_{0}, \phi=0\right)$ of a pylon located at $\phi=\phi_{w}$ involves a summation over the small angular steps of the rotation of the rotor and reads:

$$
\begin{aligned}
S_{p p}\left(r_{0}, \theta_{0}, \phi=0, \omega\right)= & \frac{B U_{X} \Delta r}{4}\left(k_{0} \rho_{0} b\right)^{2} . \\
& \int_{0}^{2 \pi} \frac{1}{\sigma^{4}} F\left(\phi, \phi_{w}\right) D_{\phi}(\alpha, \theta, \phi) . \Phi_{w w}\left(k_{r}=0, k_{X, \phi}\right) \cdot\left|\mathcal{L}^{L E}\left(k_{r}=0, k_{X, \phi}, \kappa\right)\right|^{2} d \phi
\end{aligned}
$$

where $B$ is the number of blades of the rotor ingesting the wake, $M_{x}$ is the propeller infinite upstream Mach number, $\Delta r$ is the rotor strip width, $b$ its half-chord, $\alpha$ its stagger angle and $U_{X}$ its relative velocity. $F$ is the Gaussian profile defining the pylon wake:

$$
F\left(\phi, \phi_{w}\right)=\exp \left[-a\left(\frac{\phi-\phi_{w}}{b_{w} r}\right)^{2}\right]
$$

and $D_{\phi}$ is a directivity term that depends only on the geometry:

$$
D_{\phi}(\alpha, \theta, \phi)=r_{0}^{2}(-\sin \theta \cos \phi \cos \alpha-\cos \theta \sin \alpha)
$$

Finally, $\mathcal{L}^{L E}$ is the same aeroacoustic coupling integral along the profile chord, based on Amiet's model, as was used for the rotor/rotor interaction model. The model can also use either the Von Kármán or the Liepmann spectrum.

The simplified model proved to have a computational time of 2 min30 on the CROR geometry while Kingan's full model required 40hrs on an Intel i5 $2.4 \mathrm{Ghz}$ dual core processor, with parallelization on both cores, hence a significant interest in case of big computation matrix.

Inputs to the model are the same as for the rotor/rotor interaction: the maximum rms turbulent velocity in the wake, the corresponding integral length scale and the wake half width. They are also obtained from CFD RANS simulation using elsA solver with the k- $\omega$ SST turbulence model. Here however only the pylon is modeled, allowing steady computations to be used, while neglecting the potential effect of the front rotor on the pylon wake propagation. 


\section{Assessment of the prediction methods}

\section{A. Trailing edge noise}

The method described in Section IV-4 has been applied to the rotor alone tests described in Section III. Four different rotational speeds were assessed at a constant blade pitch setting ("pitchline"): REF+100 / REF+200 / $\mathrm{REF}+300$ / REF+350. These blade speeds covered a thrust range from close to approach conditions up to slightly higher than take-off conditions. When not explicitly stated, the default models used in the following results will be Rozenberg for the pressure fluctuation spectrum, Corcos for the spanwise correlation length and fitted Del Alamo data for the convection velocity.

The first stage of the work was to assess the effect of the surface pressure spectrum model in order to select the most appropriate one. Figure 6 shows the third octave band power levels for the take-off power setting (REF+300). The same ranking as was observed on the surface spectrum in Section IV-4 is found again here. It is clear that the Rozenberg model is the only one which approaches the measured absolute level as well as the spectrum shape and peak frequency. Assuming the model is fully relevant for the CROR blade, it would mean that the strong difference between maximum friction in the boundary layer, and wall friction, would be a key driver for a high level of the surface pressure fluctuation spectrum. This model is therefore retained for the remainder of the study.

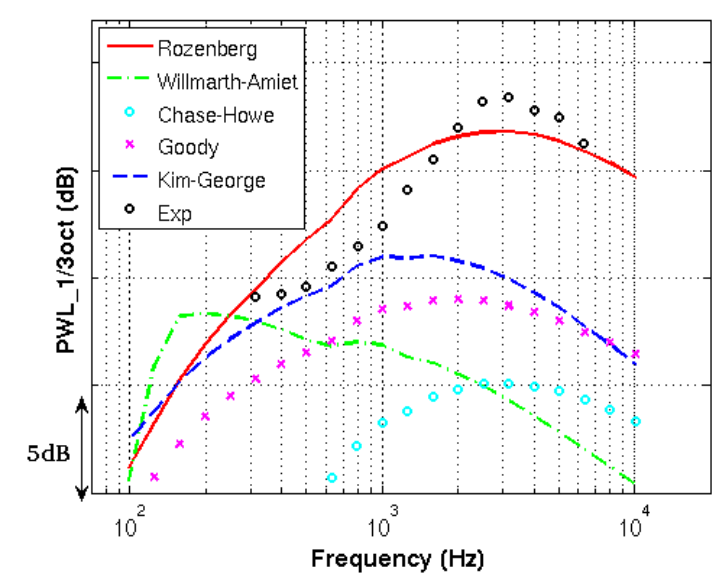

Figure 6. Effect of surface pressure fluctuation model

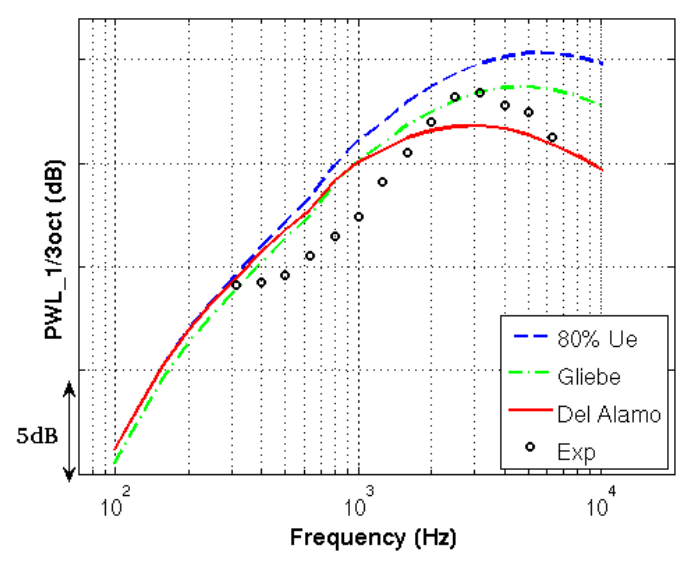

Figure 7. Effect of convection velocity

The effect of the convection velocity model is plotted in Figure 7. It appears that not accounting for the lower convection velocity of the small high frequency structures close to the wall will prevent the accurate prediction of the spectra at high frequency. The fit of the Del Alamo data seems to provide a better result, and so this model was selected.

Finally, the effect of the spanwise correlation length is investigated inFigure 8. Relative to Corcos, the Roger model shifts the peak frequency of the noise spectrum toward higher frequencies. The fact that both curves cross each other around $7 \mathrm{kHz}$ while they did not in Figure 4 is due to the use of the Del Alamo fitted convection velocity model. At high frequencies, the Del Alamo model produces reduced convection velocities that are utilised by the Corcos model, whereas the Roger model is based only on the displacement thickness. Although the Roger model is certainly more physically representative at for low frequencies, it fits the experimental data less closely. Therefore, the Corcos model was retained for the remainder of the current study, with the understanding that the low frequencies are overestimated, but that future work will concentrate on improving the model in this regime. 


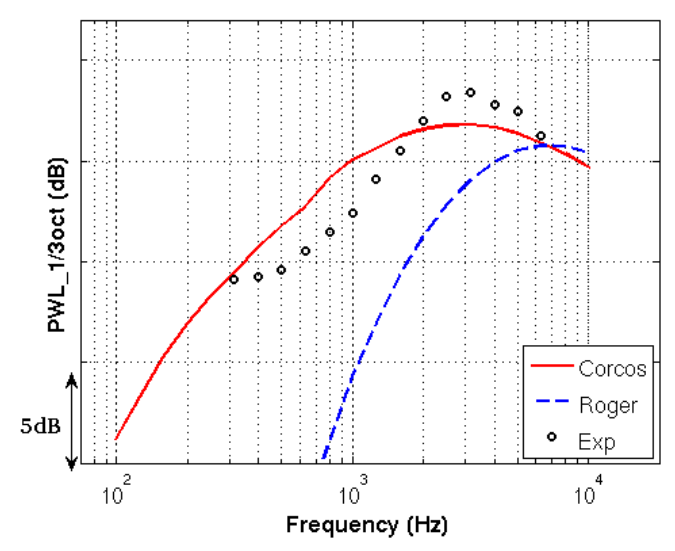

Figure 8. Effect of spanwise correlation length model

Once the empirical models were selected, the four power settings were computed in order to assess the thrust effect on the noise. Acoustic power level results are displayed in Figure 9. They show that the spectrum shape and the peak frequency are rather well predicted by the model, indicating that the method estimates the turbulence scales to a good order of magnitude. On the other hand, the thrust effect is not well assessed by BoB: while the test shows an exponent of 16.5 on the relative tip speed, the model only predicts 8.6. Only at very high tip speed is there better consistency between the model and the measurement.
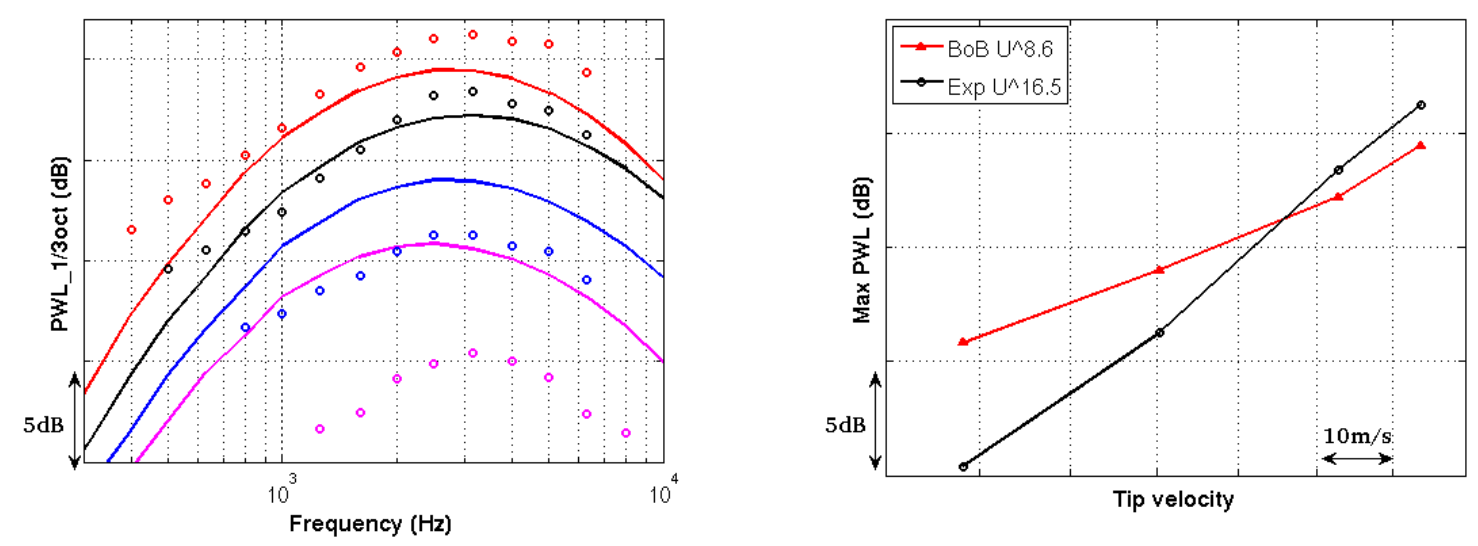

Figure 9: Thrust effect on rotor alone noise

Left: third octave band spectra - Plain: prediction, Dashed: experiment - Colours: different rotational speeds Right: Maximum PWL of the third octave band spectrum - Red: prediction, Black: experiment

This analysis has showed the difficulty associated with predicting the trailing edge noise. While there is a fairly good confidence in the CFD input data, as their use in predicting tonal noise showed excellent results ${ }^{25,26}$, the main uncertainties remain in the empirical models of the surface pressure fluctuation spectrum, convection velocity and spanwise integral length scales. It is intended to undertake more work on these topics to improve the prediction accuracy. These improvements could be achieved either from heavily instrumented experiments or via high fidelity CFD computations such as wall-resolved LES or even DNS.

Another possible explanation for the mismatch between prediction and test data is the presence of other noise sources. The most probable sources are the tip noise due to the interaction between the tip vortex and the tip edge of the blade, and perhaps the blade root noise due to the complicated 3D flow feature in the hub region caused by the hub boundary layer and the blade passage vortices. Specific models would have to be developed to investigate these sources further. 


\section{B. Rotor-wake interaction noise}

The rotor wake interaction noise method, described in section IV-B, was then applied to the CROR configuration of the rig 145 build 1 DREAM test. Again, four different power settings were analysed, with the same rotational speeds as for the single rotor configuration. As mentioned in Section III, an estimation of the interaction noise contribution in the total noise was computed by removing twice the rotor alone noise that was measured in the single rotor configuration. This approximation was justified, in part, because the single rotor pitch setting was adjusted to ensure the rotor had the same thrust as the front rotor in the CROR configuration. As a result, the blades were comparably loaded and, thus, similar trailing edge noise levels were

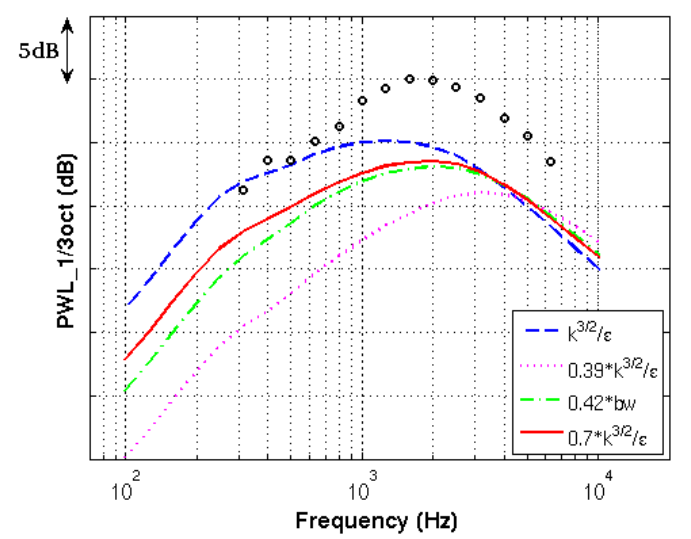

Figure 10. Effect of integral length scale model

expected from the two front rotors. There are some simplifications, to be expected, in this approach as the front and rear rotor blade designs, speeds and blade numbers were different. Nonetheless, it is believed to be a reasonable approximation which is reinforced by trailing edge noise computations that showed similar trailing edge noise levels for both rotors in the CROR configuration.

The effect of the integral length scale formula was first studied on the take-off thrust case REF+300 (Figure 10) and revealed two tendencies. A lower length scale logically shifts the spectrum peak toward higher frequencies as the size of most energetic turbulence structures is decreased, but it also significantly reduces the peak noise level by roughly $3 \mathrm{~dB}$ for a $50 \%$ reduction of the length. Surprisingly, the empirical model $(0.42 \mathrm{bw})$ is the one that best captures the peak frequency of the spectrum. Finally, an empirical coefficient of 0.7 would be the necessary for the RANS formula to obtain the same matching. As far as absolute levels are concerned, they are underestimated by about $7 \mathrm{~dB}$ for the two best matching curves. This is far from an accurate prediction.

The effect of the thrust is investigated in Figure 11. The same four rotational speeds were chosen as used for the single rotor configuration and assuming a constant $\mathrm{C}_{\Lambda}=1$. The increase of the rotational speed of the blade will increase both the relative blade speed and the incidence of the profiles. Therefore, the effect on noise will be a combination of a velocity effect and a loading effect, which explains the use of the terminology "thrust effect". Consistently with the previous discussion, spectrum shapes and peak frequencies look to be well predicted for all the speeds, while the absolute levels are all underestimated. On the other hand, the variation of the maximum power level as a function of relative tip speed appears to be reasonably predicted by the model, which would suggest that
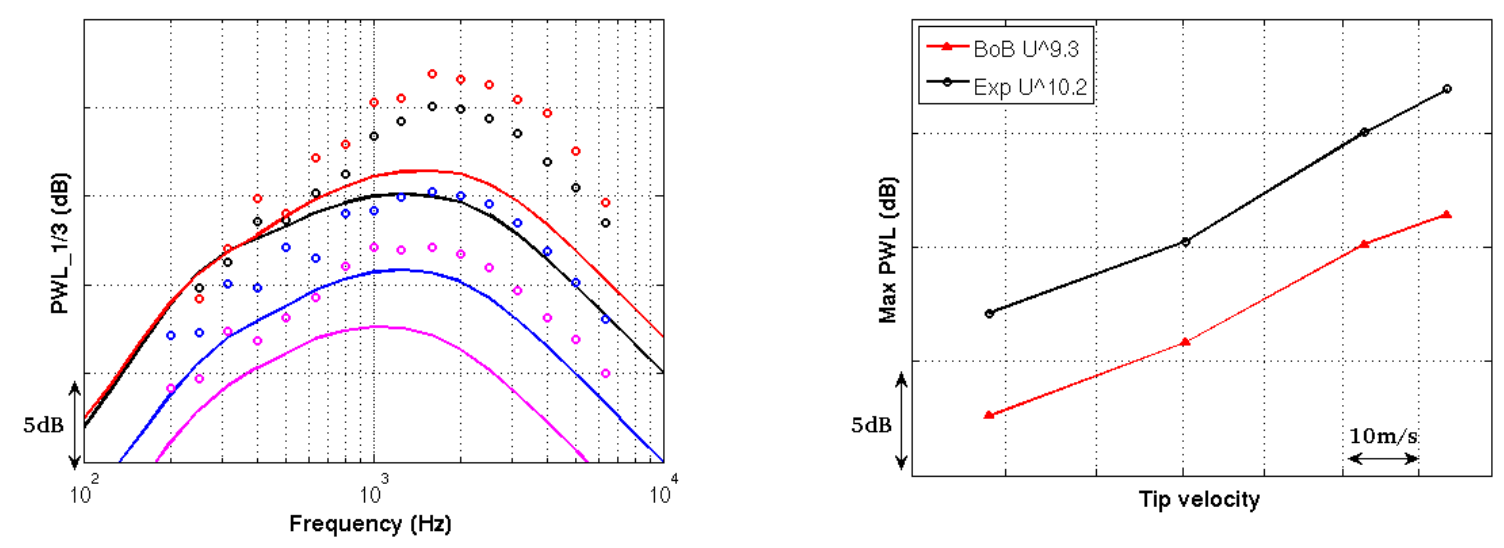

Figure 11. Thrust effect on rotor-wake interaction noise

Left: third octave band spectra - Plain: prediction, Dashed: experiment - Colours: different rotational speeds Right: Maximum PWL of the third octave band spectrum - Blue: prediction, Green: experiment 
the model embeds the correct variation of the parameter driving the noise. Assuming that the thrust effect modelling is correct, the $7 \mathrm{~dB}$ underestimation would have to be looked for in the input parameters of the model or in the measured data. The first possible cause could be an underestimation of the rms velocity or equivalently of the turbulent kinetic energy in the CFD computation. The accuracy of predictions of the wake propagation is not yet demonstrated, since no wake velocity measurements like PIV, LDV or hotwire were made during this experiment. $7 \mathrm{~dB}$ is equivalent to an error of $125 \%$ on $u_{r m s}$ or $400 \%$ on the turbulent kinetic energy in the CFD. Considering the good results obtained in the tonal noise prediction ${ }^{25,26}$ with those CFD computations, it is unlikely that such a large error exists, so CFD uncertainty could only explain a small part of the mismatch. A second significant input parameter is the wake half width, but for the same reason the error on this parameter is likely to be small. Also, the relative velocity $U_{X 2}$ that is seen by the rear rotor should be easily predicted by the CFD. Another explanation can be looked for in the accuracy of the measured noise data. Indeed, the haystacking phenomenon due to the crossing of the open jet wind tunnel shear layer by the acoustic rays causes a broadening of the tones. It prevents the accurate separation of tonal noise and broadband noise at high frequencies where tones finally disappear in the broadband noise. It is therefore known that the measured broadband level is overestimated by probably a few dBs for those frequencies. Inflow measurements would be necessary to avoid this problem, but this causes other difficulties for the measurement of broadband noise; such extensive measurement were not available during that experiment, see Parry et $\mathrm{al}^{2}$.

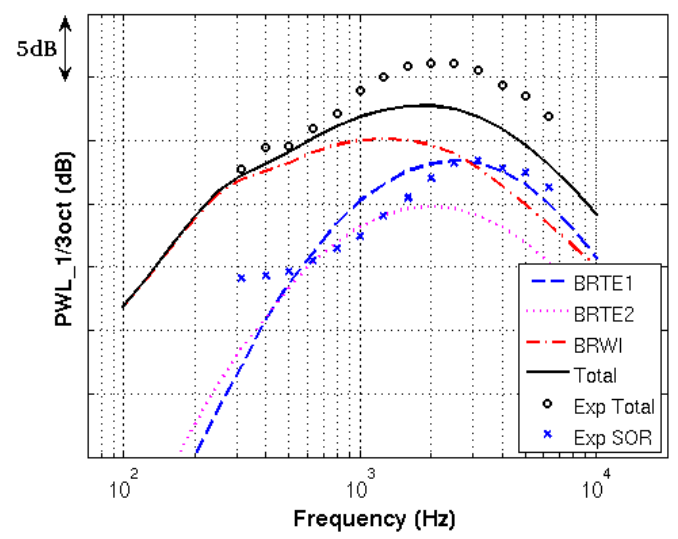

Figure 12. Breakdown of noise sources

Lines: prediction, symbols: experiment

BRTE1: front rotor trailing edge noise, BRTE2: rear rotor trailing edge noise

BRWI: rotor wake interaction noise, Exp: Experiment, SOR: single open rotor

To conclude this section, Figure 12 provides a comparison of the three computed sources for the take-off power setting (trailing edge noise of front rotor: BRTE1, trailing edge noise of rear rotor: BRTE2 and rotor wake interaction noise: BRWI), and of the total noise (computed and measured). Globally, the method shows an underestimation of $3.5 \mathrm{~dB}$ on the total noise. The level of the single rotor test (SOR) is also indicated as a reference, which confirms that the trailing edge noise of the front rotor is similar in both single rotor and CROR configurations. Figure 12 also shows that, for this configuration, the trailing edge noise appears in the experiment as a second order source relative to the rotor/rotor interaction source at low-mid frequencies. However, the trailingedge sources become more important at high frequencies, contributing to 1 or $2 \mathrm{~dB}$ to the total noise.

\section{Pylon-Wake interaction noise}

The pylon-wake interaction noise model was assessed on the installed configuration of the DREAM project wind tunnel test where a pylon was installed upstream of the front propeller. A comparison between the complete Kingan model and the newly developed simplified model, described here in section IV-C, has been performed first. Figure 13 shows for the four previous power settings that both models are very consistent for most of the spectrum, only at low frequency some differences logically appear. This demonstrates that the simplified model could be preferred for fast design iterations while the complete model would be reserved for final design evaluation. 


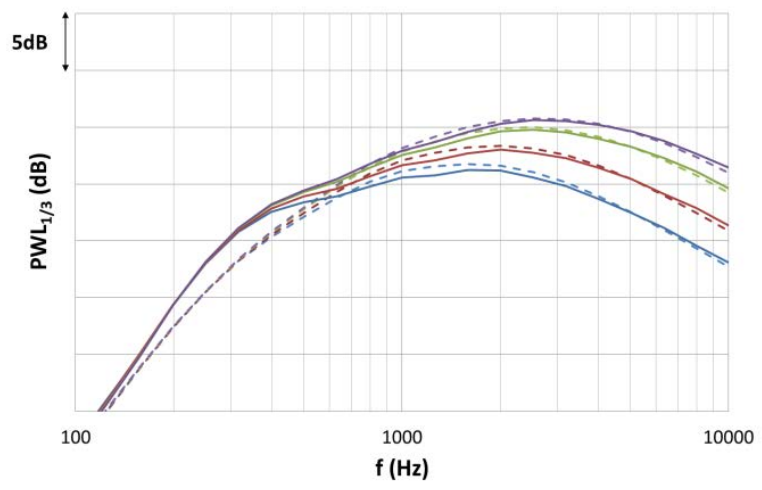

Figure 13. Pylon interaction noise model Dashed: Kingan model - Solid: simplified model Colours: different power settings

Figure 14 shows the total noise measured in isolated and installed configurations for the flyover arc, in the direction of the blade rotational velocity when the blade hits the pylon wake. Strictly speaking, the displayed parameter is not a true acoustic power; it is the integration of the acoustic intensity over the flyover polar arc and assuming uniform azimuthal directivity. This is done in order to obtain an indicator comparable to the previous sections. The noise increase due to the pylon presence is clearly visible and features strong low frequency content. Pylon induced noise is obtained by a subtraction between the two configurations, but keeping in mind that the levels above $3000-4000 \mathrm{~Hz}$ will not be reliable as there is not enough difference between the two configurations.

The comparison with the prediction is displayed in Figure 15 for the four rotational speeds. The order of magnitude of the predicted acoustic power appears to be in a similar range to the measurements. The slope between $500 \mathrm{~Hz}$ and $1500 \mathrm{~Hz}$ is reasonably predicted but the collapse starting about $1500-2000 \mathrm{~Hz}$ is less strong in BoB results. At low frequencies, the model predicts the usual drop due to the low frequency slope of the turbulence spectrum, however it can be noticed that the effect of blade speed disappears from the predictions. There, it is the experimental data that does not seem consistent, showing roughly constant noise levels. This behaviour is not usual for airfoil and rotor noise, and it is not understood so far whether this phenomenon is actual pylon/rotor noise or a facility noise due to the installed configuration.

As far as the rotational speed effect is concerned, the model predicts an increase of $5 \mathrm{~dB}$ at $1000 \mathrm{~Hz}$ and $9 \mathrm{~dB}$ in high frequencies, between the slowest and fastest rotor speeds, while the experiment shows a rather constant $10 \mathrm{~dB}$ increase.

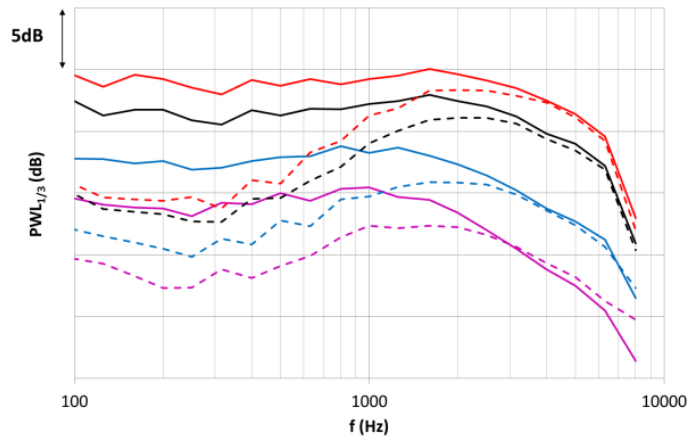

Figure 14. Installation effect (Experiment)

Plain: installed config., dashed: isolated config. Colours: different rotational speeds

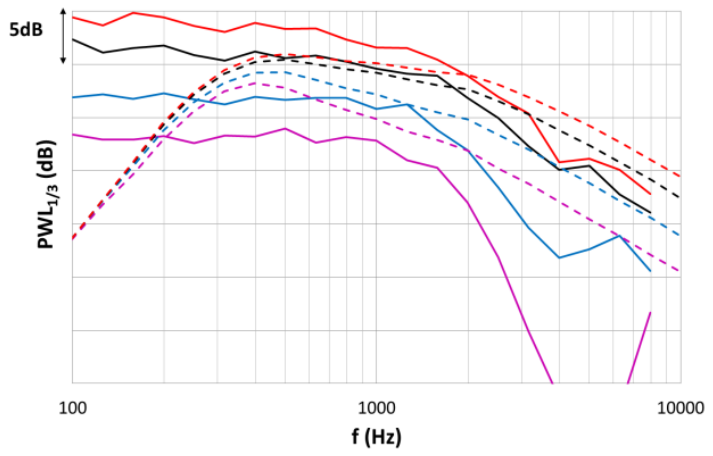

Figure 15. Installation effect (BoB vs Exp.)

Plain: experiment, Dashed: prediction Colours: different rotational speeds 


\section{Conclusion}

A comprehensive prediction method has been built and implemented for the prediction of CROR broadband noise. Comparisons have been made between the method and measurements taken on Rolls-Royce's Rig 145 build 1 wind tunnel tests, both isolated and with Airbus-designed installation features. These initial comparisons are promising but still show significant inconsistencies. Generally, the method predicts realistic spectrum shapes and peak frequencies. The absolute levels of the trailing edge noise and pylon interaction noise are found, but without accurately reproducing the thrust or rpm effect which is a critical shortfall. For the rotor-wake interaction, the situation is reversed; the levels are strongly underestimated while the thrust effect is correctly predicted.

All segments of the method shall now be reviewed to identify potential errors. CFD computations shall be more deeply investigated to identify whether there could be residual sensitivity to mesh quality, turbulence model and other numerical parameters. CFD post-processing routines shall also be audited to ensure they are robust. Further investigations are required on all the empirical models that feed the method. There is a requirement for new wellconstructed experiments that allow the various noise sources to be assessed in isolation and, for the trailing edge noise, there is a critical need on such tests for dense instrumentation close to the trailing edge, and for high fidelity boundary layer simulations. Wall resolved LES may start to reach the desired reliability (at least on canonical configurations) and could provide very valuable data to build new ad-hoc surface pressure spectrum models. For the rotor/rotor interaction noise, flow measurements such as PIV or hotwire are now mandatory to assess the accuracy of the CFD RANS parameters. Here, LES type simulations (DES...) of a partial configuration (single rotor for instance) may also provide the required information to better understand the wake turbulence.

It should also be kept in mind that only a few noise sources have been modelled. Shortfalls may be due to the presence of other sources such as blade tip noise or blade root noise, for example. 3D flow fields in those areas will continue to be investigated, along with the complex unsteady blade response effects, and new noise models will be developed.

Finally, the experimental data shall also be reviewed, in particular to estimate the impact of the haystacking effect. For this, future work will target the assessment of the methods on other wind tunnel tests performed recently within the Cleansky JTI-SFWA European project by Airbus and Rolls-Royce on the so-called Z08 rig. These tests featured both out-of-flow and inflow measurements. Additionally, several blade designs were tested so that the ability of the method to capture blade design effects will be evaluated.

\section{References}

${ }^{1}$ Hager, R. D. \& Vrabel, D., Advanced Turboprop Project, NASA SP-495, 1988

${ }^{2}$ Parry A. B., Kingan M., Tester B. J., Relative importance of open rotor tone and broadband noise sources, AIAA Paper 2011-2763, 2011

${ }^{3}$ Blandeau V., Aerodynamic broadband noise from contra-rotating open rotors, $\mathrm{PhD}$ thesis, University of Southampton, 2011.

${ }^{4}$ Blandeau V, et al., Broadband noise predictions from uninstalled contra-rotating open rotors, International Journal of aeroacoustics, vol 12(3), 245-282, 2013

${ }^{5}$ Amiet R.K., Compressibility effects in unsteady thin-airfoil theory, AIAA Journal, 12(2):252_255, 1974

${ }^{6}$ Amiet R.K., Acoustic radiation from an airfoil in a turbulent stream, Journal of Sound and Vibration, 41(4):407-420, 1975.

${ }^{7}$ Amiet R.K. . High frequency thin-airfoil theory for subsonic flow, AIAA Journal, 14(8):1076_1082, 1976.

${ }^{8}$ Amiet R.K., Noise due to turbulent flow past a trailing edge, J. of Sound and Vibration, vol 47(3), 387-393, 1976.

${ }^{9}$ Amiet R.K., Effect of the incident surface pressure field on noise due to turbulent flow past a trailing edge, J. of Sound and Vibration, vol 57(2), 305-306, 1978.

${ }^{10}$ Amiet R.K., Noise produced by turbulent flow into a propeller or helicopter rotor, AIAA journal, pp. 15(3) 307-308, 1977.

${ }^{11}$ Amiet R.K., Noise produced by turbulent flow into a rotor: Theory manual for noise calculation, NASA CR-181788, 1989.

${ }^{12}$ Schlinker R.H., Amiet R.K., Refraction and Scattering of Sound by a Shear layer, NASA CR-3371, 1980.

${ }^{13}$ Schlinker R.H., Amiet R.K., Helicopter rotor trailing edge noise, NASA CR-3470, 1981.

${ }^{14}$ Paterson R.W., Amiet R.K., Noise of a model helicopter rotor due to ingestion of turbulence. NASA CR-3213, 1979.

${ }^{15}$ FFowcs Williams, J. E., Hawkings, D. L., Sound generation by turbulence and surfaces in arbitrary motion, Phil. Trans. R. Soc. Lond. A, 264:321-342, 1969

${ }^{16}$ Howe M. S., Acoustics of fluid-structure interactions, Cambridge University Press, 1998.

${ }^{17}$ Goody M., Empirical spectral model of surface pressure fluctuations. AIAA Journal, 42(9):1788-1794, 2004.

${ }^{18}$ Kim Y.N., George A.R., Trailing-edge noise from hovering rotors. AIAA Journal, 20(9):1167-1174, 1982

${ }^{19}$ Rozenberg, Y., Roger, M., and Moreau, S., Rotating blade trailing-edge noise: experimental validation of analytical model, AIAA Journal, 48(5):951-962, 2010

${ }^{20}$ Rozenberg Y., Wall pressure spectral model including the adverse gradient effects, AIAA Journal Vol. 50(10), 2168-2179, 2012.

14

American Institute of Aeronautics and Astronautics 
${ }^{21}$ Gliebe P.R., Fan broadband self noise prediction model, AIAA paper 2002-2490, 2002

${ }^{22}$ Schloemers, Effects of Pressure Gradients on Turbulent-Boundary-Layer Wall-Pressure Fluctuations, J. Acoustical Soc. America, Vol. 42, No. 1, pp. 93-113, 1967

${ }^{23}$ Corcos G. M., The structure of the turbulent pressure field in boundary-layer flows, Journal of Fluid Mechanics, 18(3):353378, 1964.

${ }^{24}$ Cambier, L., Gazaix, M. elsA: an efficient object-oriented solution to CFD complexity, AIAA Paper, 2002-0108, 2002.

${ }^{25}$ Colin, Y., Caruelle, B., Nodé-Langlois, T., Omais, M. et Parry, A., Computational strategy for predicting CROR noise at low-speed, part 1 : review of the numerical methods. AIAA paper 2012-2221, 2012

${ }^{26}$ Colin Y., Blanc F., Caruelle B., Barrois F., Computational strategy for predicting CROR noise at low-speed Part II: investigation of the noise sources computation with the chorochronic approach, AIAA paper 2012-2222, 2012

${ }^{27}$ Ricouard et al., Installation effects on contra-rotating open rotor noise, AIAA paper 2010-3795

${ }^{28}$ Glegg, S., Baxter, S. M., and Glendinning, A. G. , The prediction of broadband noise from wind turbines, Journal of Sound and Vibration, 118(2):217-239, 1987.

${ }^{29}$ Hinze J. O., Turbulence. McGraw-Hill, 1959.

${ }^{30}$ Jurdic V., Joseph P., and Antoni J., Investigation of rotor wake turbulence through cyclostationary spectral analysis. AIAA Journal, 47(9):2022-2030, 2009.

${ }^{31}$ Spalart, P.R., Allmaras, S.R., A One-equation Turbulence Model for Aerodynamic Flows, La Recherche Aerospatiale, Vol. 1, pp. 5-21, 1994

${ }^{32}$ Menter, F. R., Two-Equation Eddy-Viscosity Turbulence Models for Engineering Applications, AIAA Journal, Vol. 32, No. 8, 1598-1605, 1994.

${ }^{33}$ Zhou, Q., Joseph, P. F., Frequency-domain method for rotor self-noise prediction, AIAA Journal, 44(6):1197-1206, 2006.

${ }^{34}$ Kingan, M., Open rotor broadband interaction noise, Journal of Sound and Vibration, 332, (17), 3956-3970, 2013

${ }^{35}$ Roger M. and Moreau S., Back-scattering correction and further extensions of Amiet's trailing-edge noise model. Part 1: theory. Journal of Sound and Vibration, 286(3):477-506, 2005.

${ }^{36}$ Roger M., Moreau S., Broadband self-noise from loaded fan blades, AIAA Journal, Vol. 42, No. 3 (2004), pp. 536-544, 2004.

${ }^{37}$ Roger M., Broadband noise from lifting surfaces, analytical modelling and experimental validation. In: Noise sources in turbulent shear flows: fundamentals and applications, CISM series 545, edited by R. Camussi, Springer, p. 289-344, 2013.

${ }^{38}$ Del Alamo J., Jimenez J. , Estimation of turbulent convection velocities and corrections to Taylor's approximation, J. Fluid Mech., vol. 640, pp. 5-26, 2009.

${ }^{39}$ Brooks T. F., Hodgson T. H., Trailing edge noise prediction from measured surface pressures. Journal of Sound and Vibration, 78(1r):69-117, 1981.

${ }^{40}$ Palumbo D, Determining correlation and coherence lengths in turbulent boundary layer flight data, Journal of Sound and Vibration, 331 3721-3737, 2012

${ }^{41}$ Efimtsov B.M., Characteristics of the field of turbulent wall pressure fluctuations at large Reynolds numbers, Sov. Phys. Acoust. 28(4), 1982.

${ }^{42}$ Pope S. B., Turbulent Flows, chapter 6. The scales of turbulent motion, Cambridge University Press, 2000.

${ }^{43}$ Fedala $\mathrm{D}$, Modélisation du bruit à large bande rayonné par un profil isolé - Application aux turbomachines, PhD thesis.

${ }^{44}$ Ganz U.W., Joppa P.D., Patten T.J., Scharf D.F., Boeing 18-inch fan rig broadband noise test. Technical report, NASA CR1998-208704, 1998.

${ }^{45}$ Peake, N., Parry, A. B., Modern challenges facing turbomachinery acoustics, Ann. Rev. Fluid Mech, 44: 227-48, 2012.

${ }^{46}$ Crighton, D. G. The Kutta condition in unsteady flow, Ann. Rev. Fluid Mech, 17: 411-45, 1985

${ }^{47}$ Robison R, A, V,, Peake, N., Propeller unsteady distortion noise, AIAA Paper 2010-3798, 2010.

${ }^{48}$ Robison R, A, V., Turbulence ingestion noise of open rotors, PhD thesis, University of Cambridge, 2011. 